\title{
Remote-controlled experiments with cloud chemistry
}

\author{
Ryan A. Skilton, Richard A. Bourne, Zacharias Amara, Raphael Horvath, Jing Jin, Michael J. Scully, \\ Emilia Streng, Samantha L. Y. Tang, Peter A. Summers, Jiawei Wang, Eduardo Pérez, Nigist Asfaw, \\ Guilherme L. P. Aydos, Jairton Dupont, Gurbuz Comak, Michael W. George and Martyn Poliakoff
}

\begin{abstract}
Developing cleaner chemical processes often involves sophisticated flow-chemistry equipment that is not available in many economically developing countries. For reactions where it is the data that are important rather than the physical product, the networking of chemists across the internet to allow remote experimentation offers a viable solution to this problem.
\end{abstract}

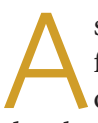

$s$ a group of 'green' chemists

from around the world, much of

our work involves inventing or

developing cleaner processes for making

known chemicals rather than synthesizing compounds for the first time ${ }^{1}$. Increasingly, such processes involve complex reactors that are expensive and difficult to obtain in some of the countries where we work. So, how can we continue to participate effectively in the international research in this area?

Three years ago, we realized something that is both obvious and important. When we are developing our processes, what we really need are the analytical data for the compounds that we make. How pure are they? What are the impurities? What is the yield of the reaction? And so on. We do not require the physical samples themselves indeed the compounds are usually sent for disposal at considerable expense as soon as the experiments are over. This realization had huge implications. If you do not physically need the compounds, then you do not need to be in the same location as the equipment - modern analytical data are electronic and are instantly transferable across the globe. So, we wondered whether it would be possible for chemists located in laboratories far away to operate continuous reactors at the University of Nottingham in the UK through the internet. Somewhat facetiously, we named this idea 'cloud chemistry'.

The idea of scientists accessing equipment remotely is certainly not new. Astronomers regularly use far-away telescopes from the comfort of their desks. Chemical engineers often operate industrial processes from a distance. Indeed, our own students here at Nottingham sometimes use the internet to check on the progress of their experiments from their homes. However, it is not common for chemists to remotely operate equipment located in someone else's laboratory without any prior training. The purpose of this Commentary is to describe how we have managed to turn the concept of cloud chemistry into a reality, to highlight some of the questions that arose, and to discuss the longer-term implications of our approach.

\section{Reactors at the ready}

For many years, chemists at Nottingham have been developing continuous reactors to carry out simple chemical transformations ${ }^{2}$ - including hydrogenation ${ }^{3,4}$, alkylation ${ }^{5,6}$ and etherification ${ }^{7,8}$ - as cleanly as possible. For nearly a decade, the reactors have been automated so that more tedious experiments, such as studying the effect of ramping up the temperature of the reactor, can be performed under full computer control $^{3}$. More recently, the automation has been taken to a higher level with the development of self-optimizing reactors that have an analytical device (chromatograph or spectrometer) downstream of the reactor that determines the composition of the product stream and quantifies the amount of each different product formed ${ }^{9-12}$. These data, which are obtained under different reaction conditions, are then fed into an algorithm that calculates a new set of reaction conditions predicted to give a higher yield of the desired product ${ }^{13-15}$. The procedure is applied iteratively to maximize the yield without the need for any intervention by the operator.

The reactors necessarily have a range of hard-wired safety trips in case the pressure or temperature exceeds pre-defined limits. The combination of these safety features with the autonomous nature of the equipment makes them relatively risk-free candidates for remote operation through the internet. The next question was simply what reaction should we try first and who was going to do it? Conveniently, the University of Nottingham has overseas campuses located in Kuala Lumpur, Malaysia, and Ningbo, China, both of which are fully integrated with the Nottingham campus in the UK. Finding someone at one of these campuses to run the first remote reaction seemed like the obvious solution and it was Jiawei Wang from the Chemical Engineering Department in Ningbo who agreed to be our guinea pig.

\section{From local to global}

The principle of a cloud chemistry experiment is shown in Fig. 1. An operator in Nottingham set the equipment up and then the experiment was in Jiawei's hands. We let him take control of the computer running the experiment in Nottingham while simultaneously communicating with him via Skype so that he understood what needed to be done to run the experiment. Once started, Jiawei could check on the progress of the reaction whenever he wanted. The Supplementary Information included with this Commentary gives details of the operating procedure and shows a screenshot from his first experiment which gratifyingly was a complete success. The chemistry itself was simple: the etherification of $n$-propanol in supercritical $\mathrm{CO}_{2}$ over a $\gamma-\mathrm{Al}_{2} \mathrm{O}_{3}$ catalyst, optimized for the formation of di- $n$-propyl ether.

After this success, Jiawei naturally wanted to do something that was more relevant to his research interests and proposed his own reaction - the catalytic isomerization of hex-1-ene. With hindsight, this proved 


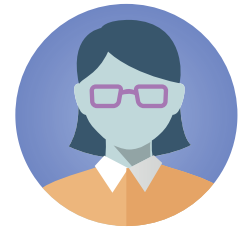

Remote operator 1
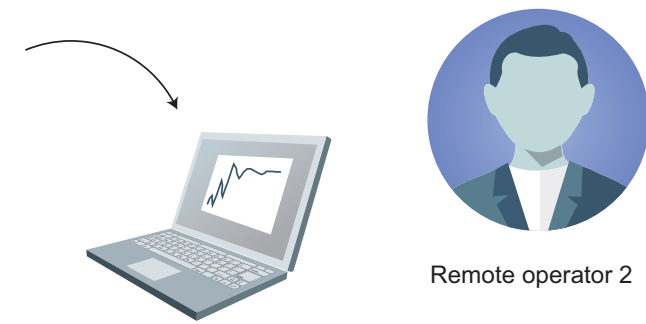

Remote operator 2

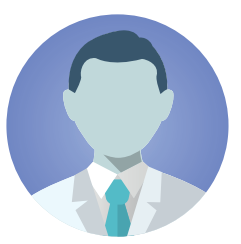

Local operator
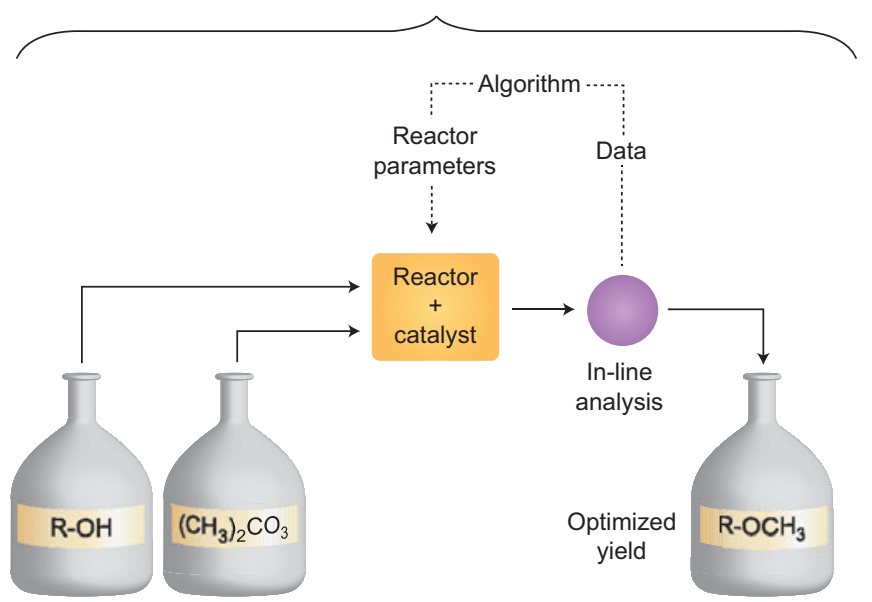

Figure 1 | The principle of cloud-chemistry experiments. At the heart of the experiments is a self-optimizing catalytic reactor, located at Nottingham University, UK. This feeds data from the in-line analysis into an algorithm to calculate new reactor parameters (for example, flow rate, temperature and so on) that are likely to give an improved yield of the desired product; in this case, a methyl ether generated by the catalytic reaction of an alcohol with dimethyl carbonate. The procedure is repeated iteratively until an optimized yield is achieved, see Fig. 2. A single computer is used to control all components of the reactor and the in-line analysis system (pumps, heaters, gas chromatograph and so on), as well as to implement the optimization algorithm. The equipment is supported by a local operator in Nottingham who refills the reactor with fresh catalyst, tops up the reactant reservoirs, primes the pumps, opens manual valves and turns on the fume cupboard in which the reactor is located. Cloud chemistry involves remote operators located across the world, any one of whom is authorized to log in to the control computer and run their own experiment in Nottingham from their home location. Once logged in, the remote operator has full control of the Nottingham computer, can activate any of the automated functions available on the reactor and can remotely access all of the data generated during the experiment. At the end of the experiment, the remote operator switches off the computer-controlled pumps and heaters, and logs out. Then, the local operator takes over, emptying the product from the collection vessel and shutting everything else down safely - descriptions of the reactor and operating procedures are given in the Supplementary Information. In the longer term, we envisage a network of different reactors located in different places, all of which would be accessible to the remote operators for cloud-chemistry experiments.

to be a mistake from the point of view of rapidly demonstrating the concept of cloud chemistry; the gas chromatograph that we were using could not resolve the isomers of hexene. Our equipment also turned out to be unsuitable for a second proposed reaction, the possible hydrogenation of $\mathrm{CO}_{2}$ to $\mathrm{MeOH}$. At this point it was decided that for subsequent trials everyone would conduct the same reaction - in this way we could focus on issues concerning the underlying principles of cloud chemistry rather than any problems associated with individual reactions. Because it was part of an ongoing research programme at Nottingham, we chose to study the etherification of $n$-butanol and $n$-pentanol with dimethyl carbonate in supercritical $\mathrm{CO}_{2}$ over different Lewis- and Brønsted-acid catalysts ${ }^{16,17}$; details of the experiments can be found in the Supplementary Information.

Following Jiawei's move to a new job, the remote-controlled experiments were successfully resumed with Peter Summers, another chemical engineer based in Ningbo. We encountered no major operational problems and the cloud-chemistry link proved to be at least as reliable as the equipment in Nottingham. At this point we were ready to expand geographically and began working with Eduardo Pérez, an assistant professor at Addis Ababa
University, Ethiopia, who had previously been a postdoc at Nottingham. The purpose of this test was to link up with a remote location where the internet connection was more temperamental than in China. Immediately we discovered that there was insufficient bandwidth for video Skype, but voice communication was fine. Moreover, because the self-optimizing reactor does not require continuous connection to the remote operator, temporary interruptions were not a problem. As before, the reactions worked well, apart from those days when it rained in Addis Ababa, which resulted in the internet connection being less reliable.

In the next step we connected with Guilherme Aydos, a PhD student working in Jairton Dupont's laboratory in Porto Alegre, Brazil. Guilherme has never been to Nottingham and is unfamiliar with the reactors we use. Except for problems with the video link, once again the experiments went very well and demonstrated that even a previously untrained operator can get good results, see Fig. 2. The final test involved Gurbuz Comak who works in the petrochemical industry in Turkey. Gurbuz was a PhD chemistry student in Nottingham, but worked in a different research group and had no prior experience of using the selfoptimizing reactors. In this particular case, the technical problem was that his company - along with many others - does not permit Skype to be used on their computer networks. To circumvent this restriction, the whole experiment was performed on a laptop connected to the $4 \mathrm{G}$ telecommunications network via a smartphone. Again, there were no problems and two experiments - including a full reaction optimization were carried out successfully.

\section{Questions about the cloud}

On a technical level, the examples described above demonstrate that our cloud-chemistry concept has not only proved itself to be feasible but also relatively easy to carry out with researchers around the world. But this initial success immediately raises a whole series of questions.

What did the remote operators think of the project? The feedback from those who controlled the reactors remotely was uniformly positive. The concept of cloud chemistry seems to have captured the imagination of all those involved and everyone has offered to participate in further experiments. It has been suggested by some that watching an optimization in progress can be quite addictive, rather like watching the bids rising during an eBay auction. One significant difference between this project and other more traditional international 
collaborations is that the barrier to getting involved at the remote site is somewhat lowered because the experiment is conducted online and can be accessed at any time of the day from any location with internet access. For example, professors and other research supervisors - who are notoriously busy and often cannot afford the time to travel to distant research facilities - could easily become involved in their students' cloud-chemistry experiments with minimal interference to their own busy schedules.

How did the Nottingham team react to the project? As shown in Fig. 1, the experiments cannot be run entirely by remote operators. Reservoirs of chemicals have to be filled, bottles of waste emptied and equipment has to be cleaned between experiments these are obviously routine tasks and not particularly exciting aspects of the research in question. The immediate incentive to the local operators was that the work being carried out was 'Nottingham chemistry' and they became just as enthused as their distant counterparts. If cloud chemistry is to become more widespread, however, one would need to think carefully about how to balance the tasks and rewards to ensure that the enthusiasm from the local team could be maintained after the initial novelty of being part of such a project subsides. In the longer term, some of the more routine tasks could perhaps be automated and the whole cloudchemistry enterprise could be supported by a specialist technician.

What are the safety issues and responsibilities? Some of the remote researchers were quite concerned by safety aspects and, in particular, by who was ultimately responsible for the experiments should anything go wrong. The answer is that this responsibility has to necessarily lie with the laboratory where the equipment is located because that is where the risks are a physical reality. Everyone has to be satisfied that the equipment is inherently safe in the sense that, whatever commands are sent to it by a remote operator, the safe operating parameters cannot be exceeded because of software controls as well as appropriate hardware safety trips. Clearly, care needs to be taken with all experiments - and especially those that use particular reactive species, such as the oxidation of organic compounds with molecular oxygen $^{18}$ - but the situation would be no different if those experiments were being undertaken by researchers in the home laboratory. As the range of chemistry widens, drawing up the risk assessments will become a joint effort between all the researchers involved in the experiment, but the ultimate go/no-go decision must always rest with the 'home team'.

\section{Were there shortcomings with the} equipment? The proof-of-concept experiments did not reveal any serious shortcomings, but the chemistry was simple and well understood. It is clear that more wide-ranging experiments would require modifications, most importantly when it comes to product analysis. The most interesting reactions are those that generate unexpected products and so the reactor needs to have a means of speciating them where necessary. An obvious solution would be to use an integrated GC-MS, but having just a single analytical technique will always be a limitation. Analysis of multicomponent mixtures, such as needed in these experiments, requires 'method development'; the appropriate instrument settings have to be established to give the desired degree of separation of the components and the instrument response must be calibrated because similar quantities of different compounds do not necessarily give similar magnitudes of response. Much of this method development can be completed by the remote operators in their home laboratory, and the instrument settings can then be transferred to the cloud-chemistry instrument. If cloud chemistry develops as we hope, there will be opportunities to incorporate multiple analytical techniques into the reactor set-up; for example, recent developments in table-top NMR spectroscopy are exciting and may prove useful in the context of these experiments.

\section{Why should people want to carry out} experiments remotely? This really is the key question. The trite answer is because they do not have the necessary equipment in their own laboratory to run experiments that could provide information that they otherwise would not be able to get. However, this is not really a sufficiently compelling reason for them to do the experiments themselves. For example, many chemists do not have local access to an X-ray diffractometer and so they send their crystals to be analysed in distant places. Even though they do not determine the structures themselves, they are usually quite happy with the results.

We believe that reaction chemistry is fundamentally different. Chemists can learn more by carrying out the reaction themselves than by outsourcing the experiment and then looking at the results, because the various reaction parameters - for example, temperature and flow rate - often interact. Therefore, additional outsourced experiments would be needed to answer questions like 'what would happen if I raised the temperature a bit more or reduced the

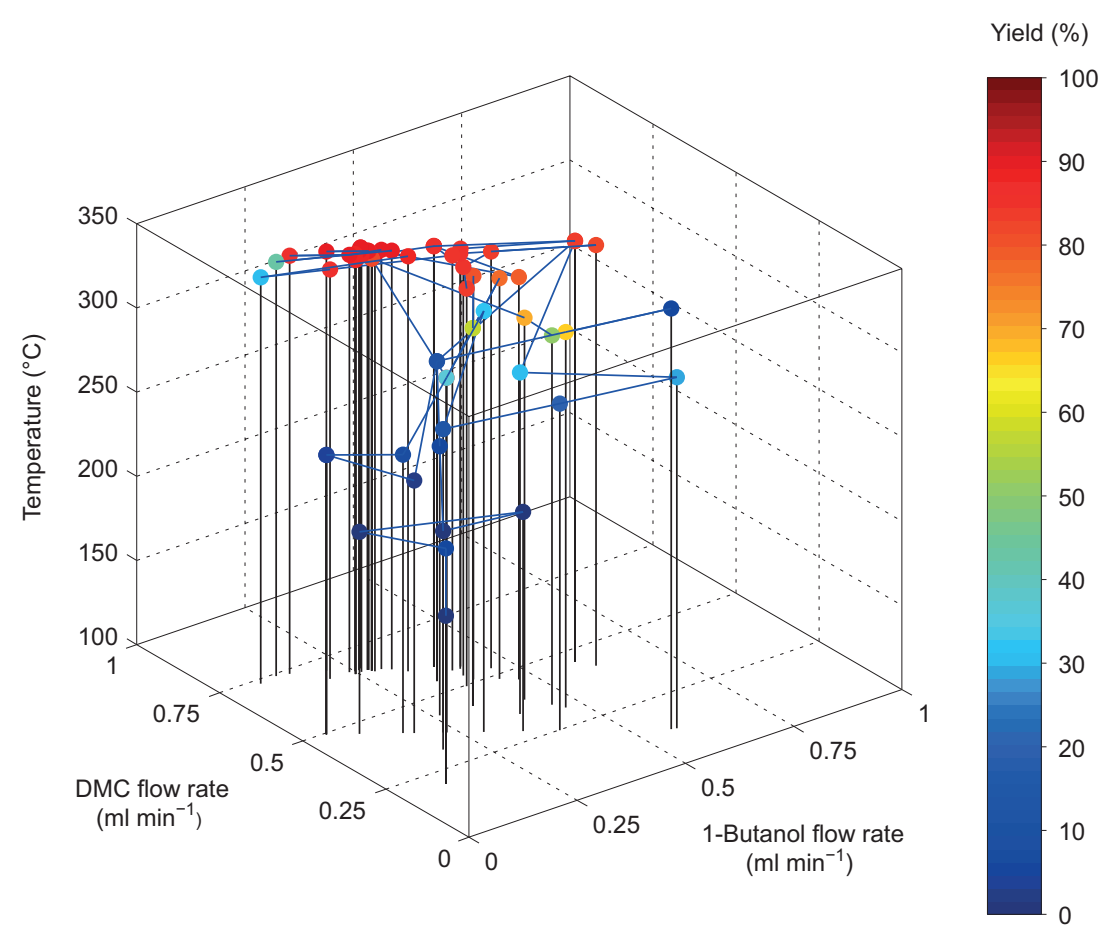

Figure 2 | The results of an optimization of the acid-catalysed reaction of 1-butanol with dimethyl carbonate (DMC) in supercritical $\mathrm{CO}_{2}$ carried out in Nottingham, UK, but controlled by Guilherme Aydos from his desk in Brazil. The colour indicates the yield of the product, methylbutylether. The whole experiment took 12 hours (see Supplementary Information for more details). 
flow rate even further?' The opportunity to perform experiments yourself might be particularly valuable with the new business model in some pharmaceutical companies where chemists sit at their desks devising new synthetic routes and then outsource the actual reactions to someone else. Think about how much more could be learned if they ran some of the reactions themselves without leaving their desks? Even in companies with active laboratories, it is becoming increasingly difficult to have a complete set of all the different types of continuous reactors that are currently available. The ability to buy time on different reactors outside their own laboratory would expand their experimental capability substantially at relatively little cost, but there are important questions concerning confidentiality and ownership of intellectual property that need to be addressed (these are discussed later in this Commentary).

The University of Nottingham has researchers spread across the globe and not only does it have an interest in maximizing the usage of the university's equipment but also, more importantly, a strong desire to promote collaborative research across its campuses. Cloud chemistry also presents an excellent opportunity for increased collaboration between skilled chemical engineers with expertise in reactor design and chemists who can recognize the new opportunities presented by novel reactors. Ultimately, we hope that cloud-chemistry experiments will be able to provide data for reactions such that a remote researcher will be able to construct a kinetic model to investigate the process in silico on their own computers without the need for further physical experiments.

How can such experiments be financed? All but the most trivial scientific experiments cost money and cloud chemistry is obviously no different. Fortunately, our proof-of-concept experiments were funded by the University of Nottingham. More generally, one could imagine two possible financial models. The first would be for the remote researchers and the home team to obtain funding for a joint research project in which the cost of cloudchemistry experiments would just be one component of the overall project budget; such projects might be supported by national funding agencies, by transnational funding such as the EU Framework Programme, and possibly by capacitybuilding schemes such as the UK Newton Fund. The second model would be a straightforward financial transaction. The remote researchers would buy a certain amount of time on the equipment to carry out their experiments, just as they might hire any other facility. One could even imagine cash-strapped research groups enticing people to contribute to a crowd-sourced project by allowing them to participate in cloud-chemistry experiments.

\section{Cybersecurity and intellectual property?}

If you authorize outsiders to have access to your computer, you could inadvertently create a link to the university network that is vulnerable to cyber-attack. This is a genuine risk. We have addressed it by ensuring that remote access is channelled through a secure gateway server with its own firewalls. Remote operators do not log in directly to the cloud-chemistry PC; instead they have usernames and passwords that allow them to enter via this gateway. In addition, the cloud-chemistry PC is isolated within the university network to prevent attack and unauthorised access to other network resources. Apparently a similar strategy is used for secure access for astronomers, for example to the Faulkes telescope project ${ }^{19}$. Intellectual property (IP) might also appear to be an important issue but cloud chemistry is no different from any other international collaborative project. You need to agree the ownership of new IP at the outset. In general, if the work were part of a joint project, one might expect the IP to be shared. On the other hand, if a company is paying to use the equipment, they might justifiably expect to keep ownership of the IP that they generate and to have details of their experiments kept confidential.

\section{What is the ultimate vision? If cloud} chemistry is ever to become more than a niche activity, many laboratories across the world need to get involved. Given the current funding atmosphere in most countries, funding agencies may see cloud chemistry as a very positive development because it has the potential to maximize access to equipment, promote industry-academic interaction and possibly accelerate the take up of new processes in industry. We foresee interesting possibilities; the first is that cloud chemistry could enable some universities to build up centres of excellence in continuousreaction technology with dedicated technicians to maintain and support the equipment - this is something that might be hard to justify for a conventional university research department.

Second, cloud chemistry could lead to a new business model and opportunities for start-ups offering such facilities. There would also be benefits for companies wishing to let potential customers try new products without prematurely disclosing their design secrets. Researchers from academia or industry could buy or be given time on a company's machines and explore their own reactions in a novel reactor or with a new catalyst without any danger that they could steal the design. This would open up a new avenue for companies to have their products test-driven and might lead to more rapid time-to-market for the finished products. In the longer term, there might be businesses that could be built-on using cloud chemistry to enable chemists to carry out experiments requiring specialized safety precautions, such as the reactions of radioactive elements, or to allow enthusiastic amateurs or pupils to carry out experiments that they could not safely attempt at home or high school.

Thirdly, a really interesting possibility involves large suppliers of laboratory chemicals. Some of these companies are already using continuous reactors to make chemicals. In principle, some of these reactors could be expanded to include cloud-chemistry facilities so that chemists could order particular chemicals and carry out the experiments without the chemicals ever having to leave the warehouse. Indeed, cloud-chemistry experiments would not necessarily have to be limited to continuous reactors because, with modern robot technology, it would be possible to carry out quite complex manipulations at a distance. Obviously cloud-chemistry experiments would cost money but there would be substantial savings because expensive packaging would not be required, there would be less need to transport hazardous chemicals around the world and less chance of them falling into the wrong hands.

In this context, cloud chemistry is very much aligned with the spirit of Chemical Leasing $^{20}$, a business model where the customer pays for the benefits obtained from the chemical, not for the actual substance itself and the consumption of chemicals becomes a cost rather than a revenue factor for the chemicals' supplier. Furthermore, such a cloud-chemistry service could have a major impact because chemists in many countries currently have to wait many months for the delivery of chemicals. With cloud chemistry, they could do their experiments almost on the day they thought of them. So, cloud chemistry could begin to remove the inequality between scientists in different countries by widening access to equipment and chemicals.

\section{What do you think?}

We have described the concept of cloud chemistry and shown that it is workable from remote countries that do not necessarily have a modern scientific infrastructure. We have discussed problems 
that might hinder the concept from becoming more widespread and have shown that none of those that we have considered are insurmountable. Finally, we have outlined a somewhat utopian vision of how this idea might develop in the future. What we are interested in now is a broader dialogue with the chemistry community. Is cloud chemistry worth pursuing or are there significant problems that we have overlooked? If you are not yet persuaded that cloud chemistry is a viable concept, what more would we need to do to convince you? We invite feedback and look forward to hearing what you think.

Ryan A. Skilton ${ }^{1}$, Richard A. Bourne ${ }^{1}$, Zacharias Amara ${ }^{1}$, Raphael Horvath ${ }^{1}$, Jing Jin ${ }^{1}$, Michael J. Scully ${ }^{2}$,Emilia Streng ${ }^{1}$, Samantha L. Y. Tang' ${ }^{1}$ Peter A. Summers ${ }^{3}$, Jiawei Wang ${ }^{3}$, Eduardo Pérez ${ }^{4}$, Nigist Asfaw ${ }^{4}$, Guilherme L. P. Aydos ${ }^{5}$, Jairton Dupont ${ }^{1,5}$, Gurbuz Comak ${ }^{6}$, Michael W. George ${ }^{1,3}$ and Martyn Poliakoff ${ }^{1 *}$.

${ }^{1}$ School of Chemistry, University of Nottingham, University Park, Nottingham NG7 2RD, UK.
${ }^{2}$ Information Services, University of Nottingham, Kings Meadow Campus, Lenton Lane, Nottingham NG7 2NR, UK. ${ }^{3}$ Department of Chemical and Environmental Engineering, University of Nottingham Ningbo China, 199 Talking East Road, Ningbo 315100, China. ${ }^{4}$ Department of Chemistry, Addis Ababa University, Ethiopia. ${ }^{5}$ Institute of Chemistry, UFRGS, Av. Bento Gonçalves 9500, 91501-970 Porto Alegre, Rio Grande do Sul, Brazil. ${ }^{6}$ ReD and Quality Assurance Department, Petkim Petrochemical Holding, M.B.12 35800, Aliaga, Izmir, Turkey.

*e-mail: Martyn.Poliakoff@nottingham.ac.uk

\section{References}

1. Poliakoff, M. \& Licence, P. Nature 450, 810-812 (2007).

2. Han, X. \& Poliakoff, M. Chem. Soc. Rev. 41, 1428-1436 (2012).

3. Hyde, J. R., Walsh, B., Singh, J. \& Poliakoff, M. Green Chem. 7, 357-361 (2005).

4. Licence, P., Ke, J., Sokolova, M., Ross, S. K. \& Poliakoff, M. Green Chem. 5, 99-104 (2003).

5. Amandi, R., Hyde, J. R., Ross, S. K., Lotz, T. J. \& Poliakoff, M. Green Chem. 7, 288-293 (2005).

6. Amandi, R., Scovell, K., Licence, P., Lotz, T. J. \& Poliakoff, M Green Chem. 9, 797-801 (2007).

7. Licence, P., Gray, W. K., Sokolova, M. \& Poliakoff, M. J. Am. Chem. Soc. 127, 293-298 (2004).

8. Gray, W. K., Smail, F. R., Hitzler, M. G., Ross, S. K. \& Poliakoff, M. J. Am. Chem. Soc. 121, 10711-10718 (1999).
9. Rasheed, M. \& Wirth, T. Angew. Chem. Int. Ed. 50, 357-358 (2011).

10. Krishnadasan, S., Brown, R. J. C., deMello, A. J. \& deMello, J. C. Lab Chip 7, 1434-1441 (2007).

11. McMullen, J. P., Stone, M. T., Buchwald, S. L. \& Jensen, K. F Angew. Chem. Int. Ed. 49, 7076-7080 (2010)

12. Moore, J. S. \& Jensen, K. F. Angew. Chem. Int. Ed. 53, 470-473 (2014).

13. Jumbam, D., Skilton, R., Parrott, A., Bourne, R. \& Poliakoff, M. J. Flow Chem. 2, 24-27 (2012).

14. Bourne, R. A., Skilton, R. A., Parrott, A. J., Irvine, D. J. \& Poliakoff, M. Org. Proc. Res. Dev. 15, 932-938 (2011).

15. Parrott, A. J., Bourne, R. A., Akien, G. R., Irvine, D. J. \& Poliakoff, M. Angew. Chem. Int. Ed. 50, 3788-3792 (2011) 16. Parrott, A. J. et al. Org. Proc. Res. Dev. 14, 1420-1426 (2010) 17. Gooden, P. N. et al. Org. Proc. Res. Dev. 14, 411-416 (2010). 18. Seki, T. \& Baiker, A. Chem. Rev. 109, 2409-2454 (2009).

19. http://www.faulkes-telescope.com/

20. http://www.chemicalleasing.com/

Acknowledgements

We thank the University of Nottingham for supporting this work. We are grateful to Professors Chris Rudd and Christine Ennew for their enthusiasm for cloud chemistry. We also thank CBMM for donation of the niobium catalysts, and all of the different sponsors that have supported the researchers in our universities who have taken part in this experiment. M.W.G. thanks the Royal Society for a Wolfson Research Merit Award.

Additional information

Supplementary information is available in the online version of the paper. 